\title{
Friction Factors for Pipe Flow of Xanthan-based Concentrates of Fire Fighting Foams
}

\author{
BOGDAN Z. DLUGOGORSKI ${ }^{1}$, TED H. SCHAEFER ${ }^{1,2}$, and \\ ERIC M. KENNEDY ${ }^{1}$ \\ ${ }^{1}$ Process Safety and Environment Protection Research Group \\ School of Engineering, The University of Newcastle \\ Callaghan, NSW 2308, Australia \\ ${ }^{2} 3 \mathrm{M}$ Specialty Materials Laboratory \\ Dunheved Circuit \\ St Mary's, NSW 2760, Australia
}

\begin{abstract}
In this paper we develop a friction factor correlation to predict the pressure drop during pumping and induction of concentrates of fire fighting foams containing around $1 \%$ of xanthan gum. Such concentrates are highly elastic, display small yield stress and exhibit significant thinning upon shearing. We demonstrate that in the turbulent regime, the Blasius equation normally used for Newtonian fluids seems to correlate well the friction factor with the Metzner-Reed Reynolds number. Our development provides an example of how the methodology used to develop the friction factor correlation can be applied to analyse a set of experimental data to verify its internal consistency. The friction factors developed in the paper can be applied to other foam concentrates that include a similar content of xanthan gum in their formulation, to predict pressure drop as a function of a flow rate and pipe diameter, provided that there exists an appropriate viscosity model. Subsequently, the paper presents experimental measurements of apparent viscosity for one foam concentrate and develops relevant viscosity models. We observe that the rheology of the concentrate is governed by the behaviour of xanthan gum. Although, the foam concentrate considered in the article is yield pseudo plastic (i.e., it follows the Herschel-Bulkley model), for the shear stresses normally encountered during pipe flow, the viscosity of the material can be described by a power law model. Over the temperature range of between 0 and $40^{\circ} \mathrm{C}$, the apparent viscosity displays only a weak dependence on temperature. Subsequent calculations of pressure drop with temperature demonstrate minor variation in pressure drop with temperature, but only in the turbulent flow regime. This suggests that induction systems intended to operate under widely varying temperature conditions should be designed to function in the turbulent flow regime.
\end{abstract}

KEYWORDS: concentrates of fire fighting foams, ATC-AFFF, AR-AFFF, class B foams, head loss in pipe flow, rheology of foam concentrates, non-newtonian fluids, Hedström number

\section{INTRODUCTION}

Concentrates of fire fighting foams must be diluted, normally to 1,3 or $6 \%$ concentration, prior to application. Most commonly, the dilution is achieved by using induction systems that siphon or pump the concentrate directly into the flowing stream of water. The proper operation of induction systems depends on concentrate viscosity, and hence on the pressure drop experienced by concentrate during its flow in the piping of an inductor. Normally, the design of an induction system would rely on experimental correlations that relate pressure drop with flow rate for a number of pipe diameters. However, there have 
been reports that these correlations may sometimes be inaccurate, resulting in the design of underperforming induction systems. From this perspective, the present study considers a set of experimental pressure drop data to develop friction factors for the flow of foam concentrates in pipes. In the process of developing the friction factors, we also verify the internal consistency of the pressure drop measurements themselves.

For the purpose of this study we selected FC600 concentrate, an alcohol resistant formulation of aqueous film forming type, which was manufactured by $3 \mathrm{M}$ Company up until 2003. The article will demonstrate that, the rheology of FC600 concentrate is governed by the presence of xanthan gum. This rheology is only slightly modified by the remaining constituents in the concentrate. Other manufacturers also produce foam concentrates that incorporate xanthan gum. In addition, new generation fire fighting foam concentrates, namely RF3 and RF6 [1], which have been recently introduced by $3 \mathrm{M}$ Company, involve xanthan gum at concentrations similar to that of FC600. This means that all three concentrates exhibit analogous rheological behaviour. For this reason, the results (especially the friction factors and the Reynolds number for the onset of turbulence) obtained in the present article possess a wide and general practical application.

In order to predict the pressure drop as a function of flow rate for non-Newtonian fluids in the laminar regime, one only needs an accurate rheological model, and then to apply the standard methodology that links the friction factor with the Reynolds number. Although a correction may be necessary for fluids characterised by yield stress, there is no effect of elasticity in the laminar regime [2]. This is not the case for predicting pressure drop in the turbulent regime, where a number of correlations exist with their predictions differing by a factor of 2 [3,4]. The agreement between the correlations deteriorates with increasing elasticity of the fluid, and decreasing the power law exponent for shear thinning fluids. This means that for strongly elastic and shear thinning fluids, such as concentrates of fire fighting foams that include xanthan gum in their formulation, at present one cannot provide reliable and accurate predictions of pressure drop in the turbulent regime. The purpose of our contribution is to redress this situation.

This article is structured as follows: The next chapter describes the material used and the experimental methodology. In the first section of the subsequent chapter, we introduce the pressure drop measurements for FC600 and then cast the data into a correlation between the friction factor and Reynolds number. A similar analysis can be applied to measurements of pressure drop of other concentrates of fire fighting foams to verify their internal consistency. Likewise, the correlation developed in that section can be applied to other foam concentrates that contain xanthan gum, to predict the pressure drop. In the subsequent section of the same chapter, we report the results from the viscometric measurements for FC600 and develop Herschel-Bulkley and power law models of viscosity. The power law models agree very well with the viscosity model derived from the pressure drop measurements, providing additional confirmation of the consistency of these measurements. The discussion chapter finishes with description of the temperature effect on the pressure drop. The major findings of this paper are summarised in conclusions.

\section{EXPERIMENTAL}

FC600 contains around 1\% of xanthan gum, and possibly other hydrocolloids, such as starch. It has a gel-like consistency. The molecular weight of xanthan gum, from 
Keltrol $^{\mathrm{TM}}$ of Kelco, included in FC600 concentrate was not determined, though it is believed to be in excess of 1,000,000 amu. The inclusion of xanthan gum increases the shelf life of the product by preventing settling of other components, adds resistance against freezing, may decrease the drag of foam solution during pumping, delays foam drainage and imparts resistance against foam destruction by polar fluids. It is for this latter reason that, the term an alcohol tolerant (or alcohol resistant) concentrate, ATC (or AR) in short, is used to denote this foam concentrate. The concentrate was stored in its container and was not treated in any way. One day before use, the concentrate was transferred to a small probe and centrifuged to remove air bubbles.

The concentrate contains ionic surfactants, both hydrocarbon and fluorocarbon based $[1,5]$, including sodium octyl and decyl sulphates, and potassium perfluoroalkyl sulfonates. These two groups of chemicals are normally present in the concentrate at levels of around 3 and $1 \%$, respectively. Salts of alkaline metals (e.g., $\mathrm{NaCl}$ ) are known to affect the rheological properties of xanthan gum solutions [6] by facilitating conformation transformations (disorder $\rightarrow$ order) of polymer chains [7-9], and we expect similar behaviour to operate for the present material as well, owing to the presence of organic salts.

Viscosity measurements were performed on a Bohlin CVO controlled stress rheometer equipped with cone and plate geometry $\left(4 \mathrm{~cm}\right.$ in diameter with the angle of $\left.4^{\circ}\right)$. The samples were pre-sheared and then subjected to shear ramps from 100 and $0.1 \mathrm{~s}^{-1}$ and then back to $100 \mathrm{~s}^{-1}$ to explore the effects of solvent evaporation and thixotropy. Several replicates were performed, including reversing the order of the shear ramp, upward (0.1 to $100 \mathrm{~s}^{-1}$ ) and then downward. No solvent evaporation was observed at lower temperatures $\left(0\right.$ and $\left.10^{\circ} \mathrm{C}\right)$. However, at higher temperatures $\left(20^{\circ} \mathrm{C}\right.$ and above), solvent evaporation led to apparent decrease in viscosity when the results from upward and downward ramps were compared. Shortening the time for each experiment by limiting the number of discrete shearing rates mitigated this effect.

No thixotropic effects were observed. This behaviour was unlike that present in the measurements reported by Korus et al. [10], who observed a significant thixotropy hysteresis in systems containing xanthan gum and starch. This difference is most likely a consequence of the presence of salts in the concentrate investigated in our study. The structure of xanthan gum solutions disrupted by shearing recovers fast in the presence of salts as opposed to slow and incomplete recovery at low salt concentrations [7].

\section{RESULTS AND DISCUSSION}

\section{Friction Factor}

Figure 1 illustrates the measurements of pressure drop of FC600 concentrate as function of flow rate and pipe diameter, for smooth Schedule 40 pipes [11]. The temperature of the measurements was not given but we expect it to be in the vicinity of $20^{\circ} \mathrm{C}$. Each plot consists of laminar and turbulent branches. The solid lines were drawn to delineate the trends. The measurements were then replotted in Fig. 2 in terms of shear stress at the wall $\left(\tau_{w}\right)$ and the shear rate at the wall $(8 V / D)$ for a Newtonian fluid, where $V$ denotes the average velocity and $D$ is the internal pipe diameter. The shear stress at the wall was computed from the pressure drop $(-\Delta p / L)$ according to 
$\tau_{w}=\frac{D}{4}\left(-\frac{\Delta p}{L}\right)$.

As expected for time independent non-Newtonian fluids [2], all laminar data trace a single line.

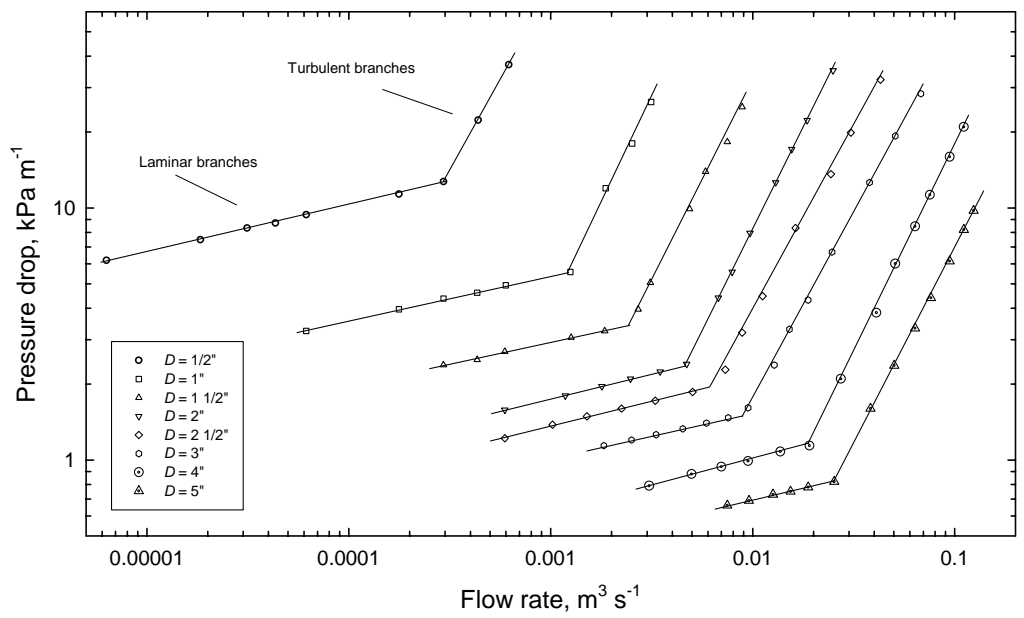

Fig. 1. The results of direct measurements of pressure drop for FC600 concentrate; replotted from Schaefer [11] in SI units.

Date for two pipe diameters (1/2" and 1 1/2") initially appeared as outliers. We hypothesised that 3/8" pipe might have been used in place 1/2" pipe, and Schedule 801 $1 / 2$ " pipe might have been employed in place of Schedule $4011 / 2$ " pipe, and corrected the internal diameters accordingly; see legend in Fig. 2. Note that the lowest shear stress in Fig. 2 is around $20 \mathrm{~Pa}$, significantly above the yield stress of the material, which is estimated to be around $5 \mathrm{~Pa}$ (next section). The second data set for FC600 concentrate provided by $3 \mathrm{M}$ Company [11] displayed pressure drops on average 2.5 lower that the measurements plotted in Fig. 1, it indicated unrealistically low wall stresses and the laminar branches could not be reduced to trace a single line. Clearly, erroneous data sets might be presently in use by industry. Consequently, this data set was removed from further consideration.

In Fig. 2, the laminar data were fitted to a power law model of viscosity

$\tau_{w}=m^{\prime}\left(\frac{8 V}{D}\right)^{n^{\prime}}$ or in more general form $\tau=m \dot{\gamma}^{n}$, where $n=n^{\prime}$ and $m^{\prime}=m\left(\frac{3 n+1}{4 n}\right)^{n}$

where $m$ and $n$ (or $m^{\prime}$ and $n^{\prime}$ ) are referred to as the fluid consistency coefficient and the flow behaviour index, respectively. The apparent viscosity is obtained from the ratio of shear stress and shear rate. For the present measurements, $m=9.91$ and $n=0.176$. Note that the flow behaviour index coincides closely with the literature data for solutions of $1 \%$ of xanthan gum [12], indicating that other components of the concentrate have relatively minor effect on the material viscosity. 


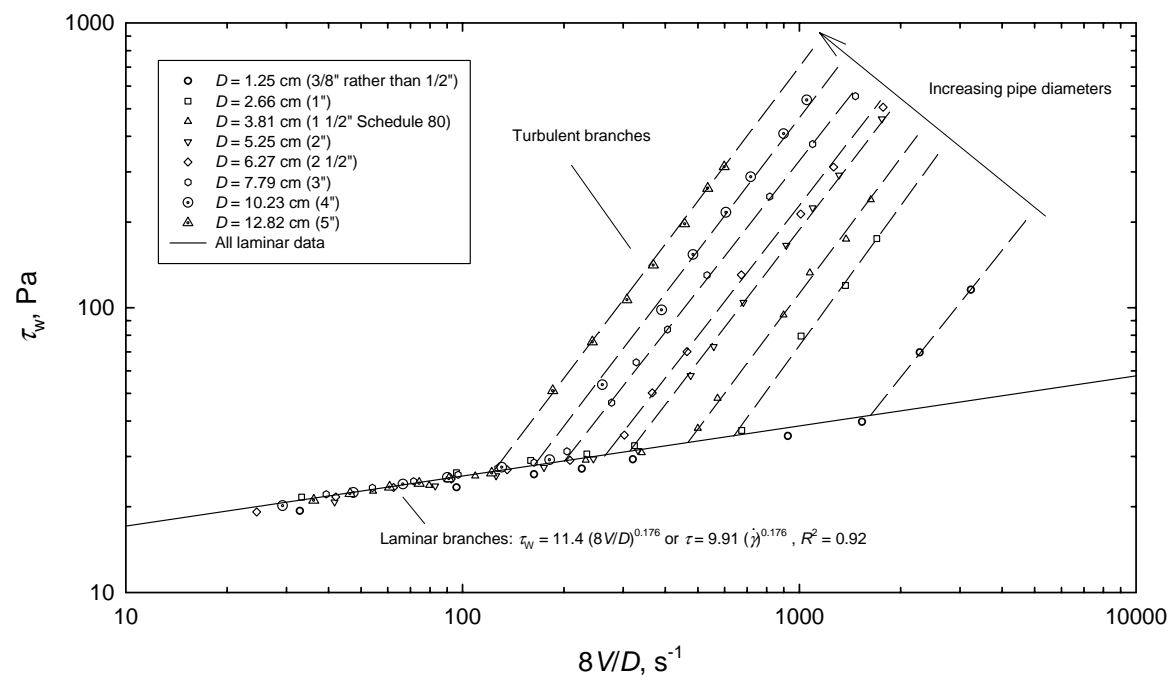

Fig. 2. The relationship between $\tau_{w}$ and $8 V / D$ for all data illustrated in Fig. 1.

Let us now introduce the definitions of the Fanning friction factor $f$ and the so-called Metzner-Reed Reynolds number $\mathrm{Re}_{M R}[13]$

$$
f=\frac{\tau_{w}}{\frac{1}{2} \rho V^{2}}, R e_{M R}=\frac{\rho V D}{\eta_{\text {eff }}} \text {, where } \eta_{\text {eff }}=\frac{\tau_{w}}{\frac{8 V}{D}}=m^{\prime}\left(\frac{8 V}{D}\right)^{n^{\prime}-1}
$$

where $\rho$ (1030 $\mathrm{kg} \mathrm{m}^{-3}$ for FC600) carries its standard meaning of density and $\eta_{\text {eff }}$ is the effective apparent viscosity. Figure 2 can now be replotted in terms of the friction factor and the Metzner-Reed Reynolds number with the results illustrated in Fig. 3.

As expected, the laminar branches follow the classical relationship of $f=16 / \mathrm{Re}_{M R}$, which applies to both viscous and elastic fluids [2]. Clearly, foam concentrates containing xanthan gum which is know for its significant elasticity $[6,8,14]$ follow this relationship as well. There appears to be no well-articulated transitional behaviour between laminar and turbulent regimes, with an abrupt transition at around $\mathrm{Re}_{M R}=1190$. The turbulent branches follow the classical Blasius expression, normally associated with Newtonian fluids

$$
f=\frac{0.0795}{R e_{M R}^{1 / 4}}
$$

The reason for this early and abrupt transition to turbulence is unclear. Direct experimental observations are needed to resolve this uncertainty. The most commonly used correlation of Dodge and Metzner [15] predicts substantially lower friction factor and a significantly higher Reynolds number for the onset of turbulent flow, as indicated in Fig. 3. Of the published correlations, only the model of Tomita [16] gives predictions similar to that of Eq. 4. At lower concentrations, in the order of 500 to 2000 ppm, 
xanthan gum acts as a drag suppressor [14]; i.e., less concentrated solutions of xanthan gum, than those studied in this paper, display very different behaviour in the turbulent regime.

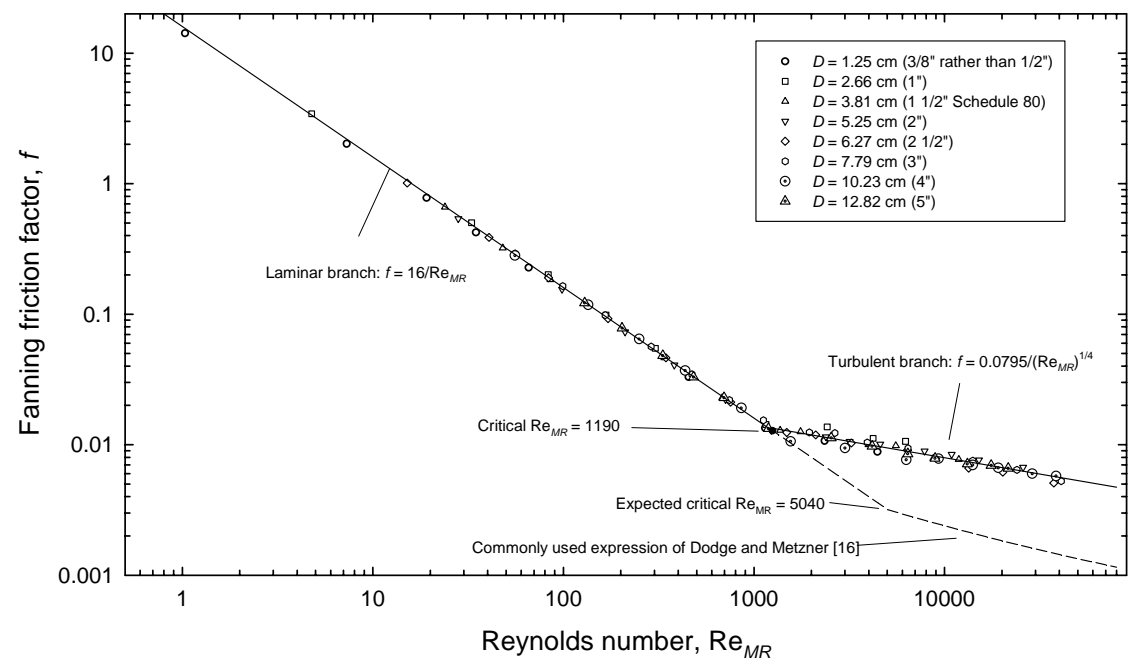

Fig. 3. Friction factor plot for FC600, as an example of a fire foam concentrate with rheological behaviour determined by its content of xanthan gum.

The methodology outlined in the current section could be applied: (i) to verify the internal consistency of the direct measurements of pressure drop; or (ii) to predict the pressure drop as function of flow rate and pipe diameter, if a viscosity model is available from viscometric measurements. Consequently, the next section develops viscosity models for foam concentrates from rotational viscometry. These models then allow us to study the effect of temperature on pressure drop, as illustrated in the last section of the present manuscript.

\section{Viscometric Measurements}

Figure 4 presents the shear - shear rate measurements for FC600 collected at temperatures between 0 and $40^{\circ} \mathrm{C}$. For clarity, no replicates are illustrated in the figure and the data represent only the first (i.e., downshear) part of the shearing loop. The power law models fitted to the measurements of Fig. 4 are listed in the second column of Table 1; see row 1 for definition of the models. Other researchers obtained viscosity models that are characterised by similar model parameters $[12,17,18]$. Note that at the concentration of xanthan gum used in foam concentrates, the power law behaviour is expected to hold for shear rates well above $1000 \mathrm{~s}^{-1}$; that is, for shear rates normally encountered in pipe flow (Fig. 2). Shear rates in excess of $100 \mathrm{~s}^{-1}$ were not accessible with the cone-plate geometry owing to the centrifugal force that engendered the material to be ejected from the narrow gap. 


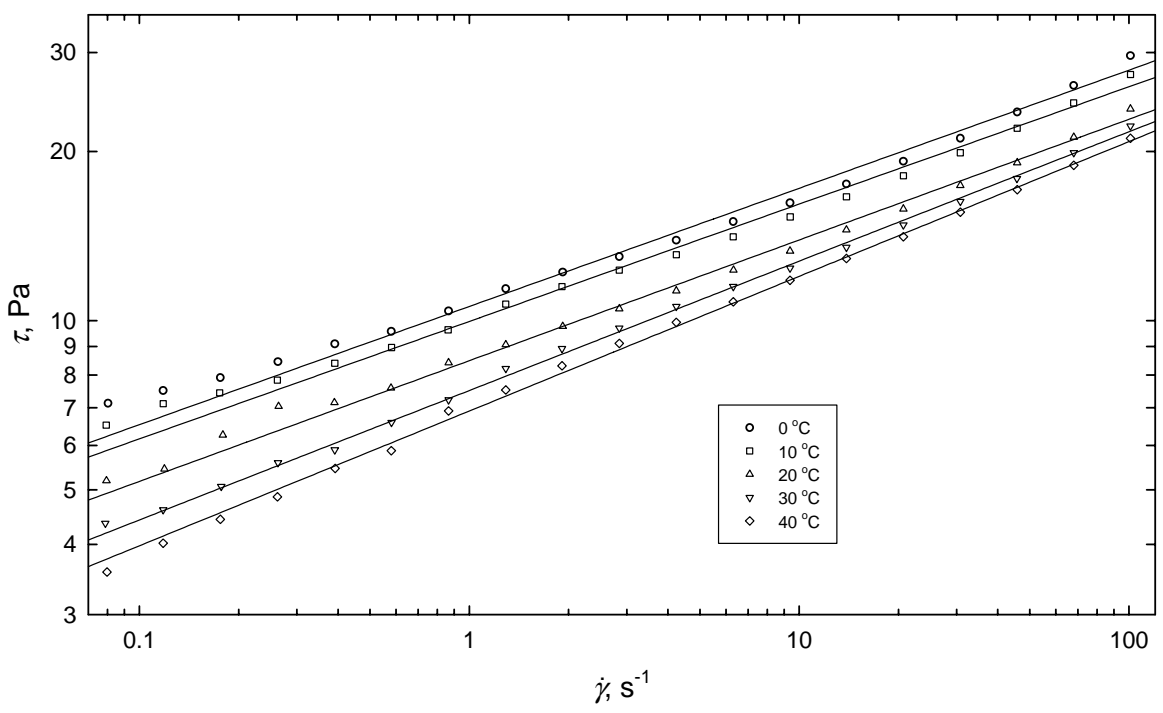

Fig. 4. Fits of power law model to the experimental data. The measurements represent the results of a single set of experiments.

Figure 4 indicates the existence of yield stress, especially at low temperatures. This observation follows from the experimental points exhibiting a levelling effect at low shear rates. During the experiments we observed that at lower values of shear stress, the cone did not rotate uniformly. Rather it moved by a succession of slip-stick steps. We interpret this behaviour as a reflection of the appearance of yield stress, with the concentrate flowing briefly only when the critical strain was exceeded. Consequently, we also derived the power law parameters by basing the models on the measurements that did not show the slip-stick phenomenon. The results are included in the third column of Table 1.

Figure 5 illustrates the application of the Herschel-Bulkley model to the present measurements for shears above the appearance of the slip-stick behaviour, with the model parameters listed in the fourth column of Table 1. Note that the apparent yield stress $\left(\tau_{o}\right)$ is merely a model parameter [19] that can only be interpreted to represent an approximate value of the true yield stress of the material. Indeed, there are reports in the literature of unphysical (i.e., negative) values of the apparent yield stress when experimental data were fitted to the Herschel-Bulkley model $[20,21]$. However, this was not the case for the current results.

The estimate of the yield stress allows us to calculate the Hedström number that gauges the importance of yield stress in pipe flow

$H e=\frac{D^{2} \rho}{m}\left(\frac{\tau_{o}}{m}\right)^{\frac{2-n}{n}}$

with the numerical values of $\tau_{o}, m$ and $n$ included in the last column of Table 1 . For the largest pipe diameter considered in this article (Fig. 1), the Hedström number varies 
between 5 and 500 , for 40 and $0^{\circ} \mathrm{C}$, respectively. These values are too small for the yield stress to affect the pressure drop [16] in the range of stresses that are of practical interest for pumping concentrates of fire fighting foams. This conclusion is also supported by the observation that in the laminar regime, the friction factor does not deviate from $16 / \mathrm{Re}_{M R}$, and by the observation that velocity profiles for viscoplastic and pseudoplastic models of the fluid are within $1 \%$ of each other provided that the flow behaviour index is sufficiently small [13], as is the case in the present study. Consequently, in further considerations, the behaviour of the concentrate was approximated by a power law model.

Table 1. Power law and Herschel-Bulkley parameters for FC600 for various temperatures; stress in Pa, shear rate in $\mathrm{s}^{-1}$. The models in the third and fourth columns were based on the data with shear stresses above the apparent yield stress (Fig. 5).

\begin{tabular}{|c|c|c|c|}
\hline $\begin{array}{c}\text { Temp } \\
{ }^{\circ} \mathrm{C}\end{array}$ & $\begin{array}{c}\text { Power Law } \\
\tau=m \dot{\gamma}^{n}\end{array}$ & $\begin{array}{c}\text { Power Law } \\
\tau=m \dot{\gamma}^{n}\end{array}$ & $\begin{array}{c}\text { Herschel-Bulkley } \\
\tau=\tau_{o}+m \dot{\gamma}^{n}\end{array}$ \\
\hline 0 & $\tau=10.5 \dot{\gamma}^{0.217}$ & $\tau=9.02 \dot{\gamma}^{0.254}$ & $\tau=9.15+2.57 \dot{\gamma}^{0.450}$ \\
\hline 10 & $\tau=9.91 \dot{\gamma}^{0.213}$ & $\tau=8.94 \dot{\gamma}^{0.239}$ & $\tau=7.42+3.24 \dot{\gamma}^{0.395}$ \\
\hline 20 & $\tau=8.49 \dot{\gamma}^{215}$ & $\tau=7.92 \dot{\gamma}^{0.234}$ & $\tau=5.53+3.45 \dot{\gamma}^{0.360}$ \\
\hline 30 & $\tau=7.51 \dot{\gamma}^{0.230}$ & $\tau=7.29 \dot{\gamma}^{0.238}$ & $\tau=5.30+3.15 \dot{\gamma}^{0.364}$ \\
\hline 40 & $\tau=6.91 \dot{\gamma}^{0.240}$ & $\tau=6.95 \dot{\gamma}^{0.238}$ & $\tau=3.37+3.38 \dot{\gamma}^{0.313}$ \\
\hline $\begin{array}{c}\text { Overall } \\
\text { model }\end{array}$ & $\tau=m^{11} e^{\frac{E}{R T}} \dot{\gamma}^{n}$ & $\tau=m^{\prime \prime} e^{\frac{E}{R T}} \dot{\gamma}^{n}$ & \\
\hline
\end{tabular}

${ }^{1} E$ denotes the activation energy in $\mathrm{J} \mathrm{mol}^{-1}, R$ is the universal gas constant in $\mathrm{J} \mathrm{mol}^{-1} \mathrm{~K}^{-1}$, and the absolute temperature $T$ is given in $\mathrm{K}$. 


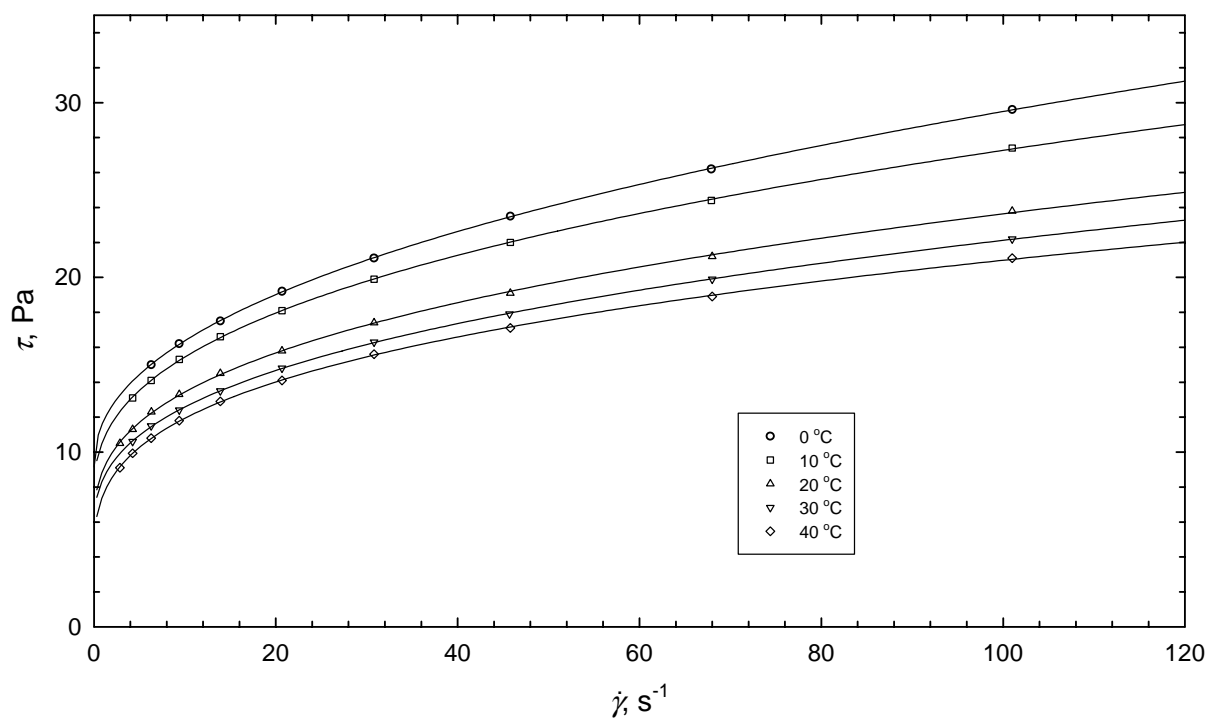

Fig. 5. Fits of Herschel-Bulkley model to the viscometric data, for stresses above the onset of the stick-slip behaviour.

Columns two and three of Table 1 demonstrate that the fluid consistency coefficient decreases significantly with temperature, whereas the flow behaviour index remains approximately constant. The temperature dependence of fluid consistency coefficient has been modelled by an Arrhenius expression [19]

$m=m^{\prime \prime} e^{\frac{E}{R T}}$

with the fit illustrated in Fig. 6, and with the master curves shown in the bottom row of Table 1.

A rather small activation energy of $5 \mathrm{~kJ} \mathrm{~mol}^{-1}$ indicates relative insensitivity of the concentrate's viscosity to temperature. For comparison, water displays the activation energy for viscosity of around 16 and glycerol of around $60 \mathrm{~kJ} \mathrm{~mol}^{-1}$. The activation energy and the present study corresponds closely with similar data for the solutions of xanthan gum published in the literature [17] indicating that the rheology of FC600 concentrate is indeed dominated by the rheology of xanthan gum. 


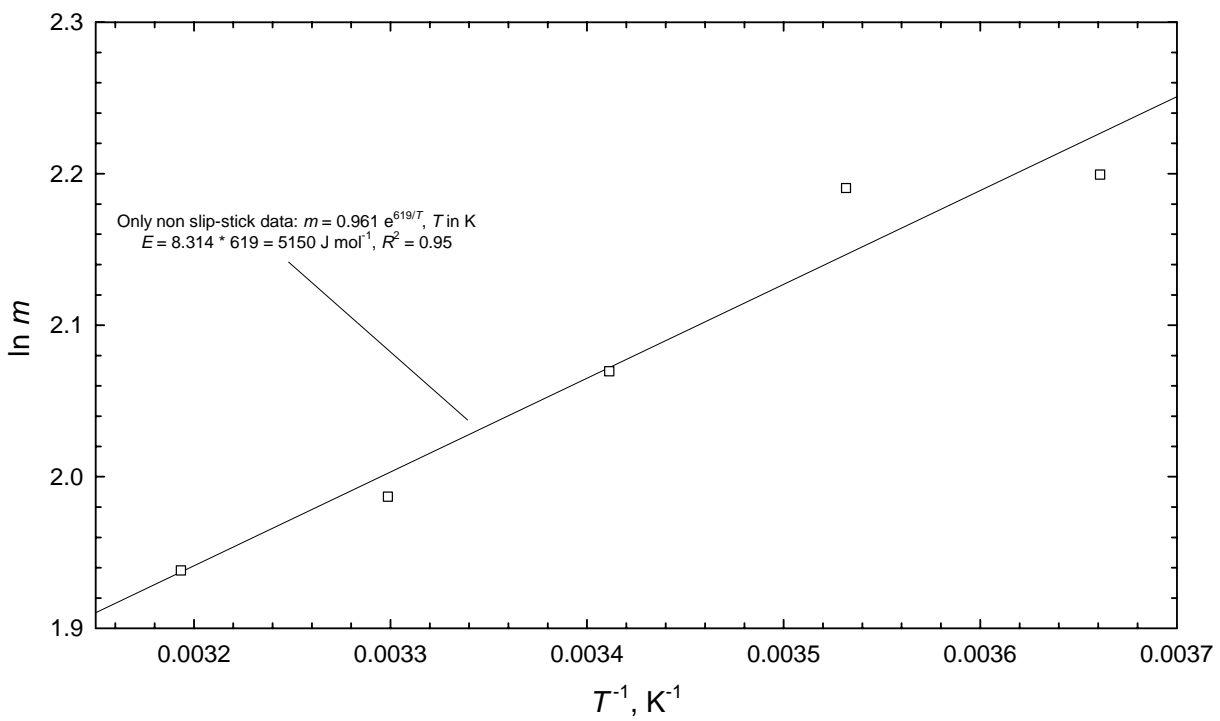

Fig. 6. Arrhenius plot to determine the activation energy for viscosity of FC600 between 0 and $40^{\circ} \mathrm{C}$.

\section{Effect of Temperature}

Figure 7 illustrates the effect of temperature on pressure drop at various flow rates and pipe diameters. All data in the figure are for Schedule 40 pipes and the inside diameters of the pipes are indicated in the figure as well to avoid confusion. The calculations performed to obtain the numerical values for developing the plots for inclusion in Fig. 7 were obtained by employing the power law models of Table 1 (third column) in conjunction with Eqs. 2 to 4 and then by expressing the results in terms of pressure drop as a function of the flow rate. It is immediately evident that even small variation of viscosity with temperature has noticeable influence on pressure drop in the laminar regime. For example, in this regime, for an induction system designed to operate at $40^{\circ} \mathrm{C}$ but used at $0^{\circ} \mathrm{C}$, the pick up of foam concentrate for mixing with water may decrease by more than three times. However in the turbulent regime, the temperature has a negligible effect on pressure drop. This means that induction systems expected to operate effectively at different temperature should be designed in the turbulent regime.

\section{CONCLUSIONS}

This paper has demonstrated that the rheology of concentrates of fire fighting foams incorporating xanthan gum in their formulations is governed by the rheology of this additive. The concentrates possess yield stress and display shear thinning; i.e., they are yield pseudoplastic materials. The yield stress, as estimated from the Herschel-Bulkley model, varies between 3 and $9 \mathrm{~Pa}$, at 40 and $0^{\circ} \mathrm{C}$, respectively. For practical applications of concentrate pumping, this yield stress can be neglected, with the viscosity of the concentrates being quite accurately described by a power law model. This simplification is also supported by the calculations of the Hedström number $(<500)$ and the existence of higher shear stresses (>20 Pa) during concentrate pumping. 
It has been argued that the pressure drop - flow rate correlations should be verified for their internal consistency prior to their applications to designing the induction systems. This can be accomplished by replotting the correlations in term of $\tau_{w}$ versus $8 \mathrm{~V} / \mathrm{D}$ to investigate whether all laminar measurements trace a single line. It turns out that for xanthan gum solutions considered in this paper, a power law model can be fitted to this line, agreeing well with the results of the viscometric measurements. Further conversion of the measurements to a relationship between a friction factor and the Metzner-Reed Reynolds number should also collapse the turbulent data into a single correlation.

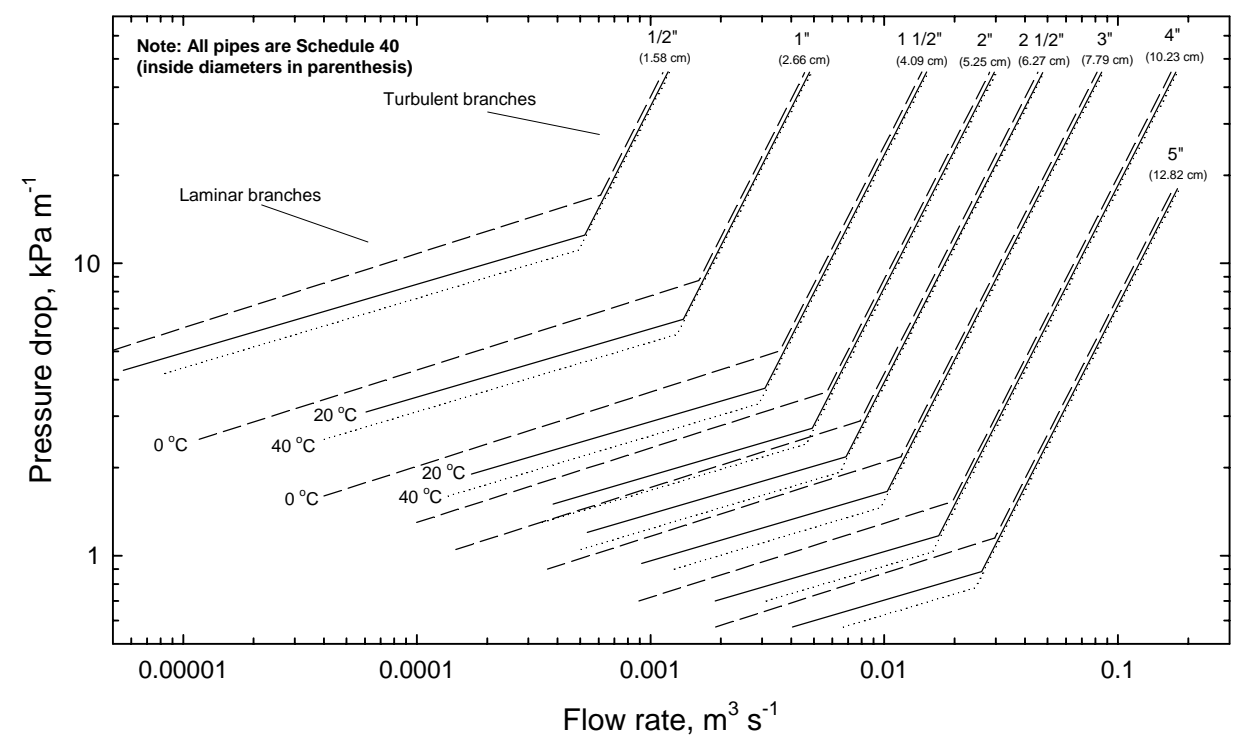

Fig. 7. The effect of temperature on pressure drop for pumping FC600 concentrate in smooth Schedule 40 pipes.

It has been shown that in the laminar regime, and for $\tau_{w}>20 \mathrm{~Pa}$, the friction factor shows no effect of yield stress and can be correlated with the Metzner-Reed Reynolds number by the classical expression of $f=16 / \mathrm{Re}_{M R}$. In the turbulent regime, the friction factor follows the Blasius correlation of $f=0.0795 / \mathrm{Re}_{M R}{ }^{1 / 4}$. The transition from laminar to turbulent flow occurs at $\operatorname{Re}_{M R}=1190$. For known viscosity models of similar foam concentrates (i.e., concentrates involving xanthan gum in their formulations), these correlations provide a means to calculate the pressure drop for given flow rate and pipe diameter, for an immediate application in design calculations.

\section{ACKNOWLEDGEMENTS}

The authors wish to thank Professor George Franks of the University of Newcastle for helpful discussions about viscometric measurements. The authors acknowledge the support of this work by 3 M Company.

\section{REFERENCES}

[1] Schaefer, T.H., Aqueous Foaming Composition, PCT/US02/38937, WO 03/049813 A1, World Intellectual Property Organization, 2003. 
[2] Chhabra, R.P., and Richardson, J.F., Non-Newtonian Flow in the Process Industries: Fundamentals and Engineering Applications, ButterworthHeinemann, Oxford, 1999.

[3] Garcia, E.J., and Steffe, J.F., Journal of Food Process Engineering, 9, pp. 93120, (1987).

[4] Heywood, N.I., and Cheng, D.C.H., Transactions of the Institute of Measurement and Control, 6, Iss. 1, pp. 33-45, (1984).

[5] Dlugogorski, B.Z., Kennedy, E.M., Schaefer, T.H., and Vitali, J., "What Properties Matter in Fire-fighting Foams?” Proceedings of $2^{\text {nd }}$ NRIFD Symposium, Tokyo, 1999, pp. 57-78.

[6] Whitcomb, P.J., and Macosko, C.W., Journal of Rheology, 22, Iss. 5, pp. 493505, (1978).

[7] Rochefort, W.E., and Middleman, S., Journal of Rheology, 31, Iss. 4, pp. 337369, (1987).

[8] Lee, H.C., and Brant, D.A., Biomacromolecules, 3, pp. 742-753, (2002a).

[9] Lee, H.C., and Brant, D.A., Macromolecules, 35, pp. 2223-2234, (2002b).

[10] Korus, J., Juszczak, L., Witczak, M., and Achremowicz, B., International Journal of Food Science and Technology, 39, pp. 641-652, (2004).

[11] Schaefer, T.H., FC600: Pressure Drop versus Flow Rate, Personal Communication, 2004, 2 p.

[12] Savage, R.M., Food Hydrocolloids, 14, pp. 209-215, 2000.

[13] Metzner, A.B., and Reed, J.C., AIChE J, 1, pp. 434-440, (1955).

[14] Bewersdorff, H.W., and Singh, R.P., Rheologica Acta, 27, pp. 617-627, (1988).

[15] Dodge, D.W., and Metzner, A.B., AIChE J, 5, p. 189, (1959); note the arrata in AIChE J, 8, p. 143, (1962).

[16] Tomita, Y., Bulletin of Japanese Society of Mechanical Engineers, 2, Iss. 5, pp. 10-16, (1959).

[17] Marcotte, M., Hoshahili, A.R.T., and Ramaswamy, H.S., Food Research International, 34, pp. 695-703, (2001).

[18] Whitcomb, P.J., and Macosko, C.W., "Rheology of Xanthan Gum Solutions," Chapter 12 in Extracellular Microbial Polysaccharides, P. Sandford and A. Laskin editors, ACS Symposium Series 45, Washington DC, 1977, pp. 160-174.

[19] Carreau, J.P., De Kee, D.C.R., and Chhabra, R.P., Rheology of Polymeric Systems: Principles and Applications, Hanser Publishers, Munich, 1997.

[20] García-Ochoa, F., and Casas, J.A., The Chemical Engineering Journal, 54, pp. B41-B46, (1994).

[21] Hannote, M., Flores, F., Torres, L., and Galindo, E., The Chemical Engineering Journal, 45, pp. B49-B56, (1991). 